\title{
The cytomegalovirus protein US31 induces inflammation through mono-macrophages in systemic lupus erythematosus by promoting NF-KB2 activation
}

Gangqiang Guo ${ }^{1}$, Sisi Ye', Shangdan Xie², Lele Ye ${ }^{1}$, Cong Lin², Min Yang ${ }^{2}$, Xinyu Shi ${ }^{1}$, Fangyan Wang ${ }^{3}$, Baoqing Li ${ }^{4}$, Ming $\mathrm{Li}^{5}$, Chaosheng Chen ${ }^{6}$, Lifang Zhang ${ }^{1}$, Huidi Zhang ${ }^{6}$ and Xiangyang Xue ${ }^{1}$

\begin{abstract}
It has been hypothesized that human cytomegalovirus (HCMV) infection, especially in monocyte and CD34 (+) myeloid cells, acts as a important regulator of immune system to promote inflammation in multiple autoimmune diseases. The aim of this study was to elucidate the HCMV gene expression profiles in the peripheral blood mononuclear cells (PBMCs) of SLE patients and demonstrate the effect and mechanism of viral gene associated with SLE in mono-macrophages functions. Using two RNA-Seq techniques in combination with RT-PCR, 11 viral genes mainly associated with latent HCMV infection were identified in the PBMCs of SLE patients. Among these viral genes, US31 with previously unknown function was highly expressed in the PBMCS of SLE patients compared to healthy controls. Analysis of function indicated that US31 expression could induce inflammation in monocyte and macrophage and stimulate macrophage differentiation toward an M1 macrophage phenotype. Screening via protein chips in combination with bioinformatic analysis and consequent detection of mono-macrophages function indicates that the direct interaction between US31 and NF-KB2 contributed the NF-kB2 activation. Consequent analysis indicated US31 directly interacted with NF-KB2, contribute to the polyubiquitination of the phosphorylated p100 and consequent activation of NF-KB2. Taken together, our data uncovered a previously unknown role of the HCMV protein US31 in inducing NF-KB-mediated mono-macrophage inflammation in the pathogenesis and development of SLE. Our findings provide a foundation for the continued investigation of novel therapeutic targets for SLE patients.
\end{abstract}

\section{Introduction}

Increasing evidence suggests that human cytomegalovirus (HCMV), which commonly infects human populations, constitutes an important trigger of SLE and can further aggravate disease progression ${ }^{1-3}$. In turn, systemic lupus erythematosus (SLE) aggravation also promotes

\footnotetext{
Correspondence: Huidi Zhang (hdzhang@163.com) or

Xiangyang Xue (wzxxy001@163.com)

'Department of Microbiology and Immunology, Institute of molecular virology and immunology, Institute of Tropical Medicine, Wenzhou Medical University, Wenzhou, China

${ }^{2}$ Second Clinical College, Wenzhou Medical University, Wenzhou, China Full list of author information is available at the end of the article

Gangqiang Guo and Sisi Ye contributed equally to this work.

Edited by A. Oberst
}

HCMV infection ${ }^{4}$, resulting in a vicious cycle. However, there is currently very little information available regarding the mechenisms after HCMV infection with the immunological function and SLE morbidity.

It has been demonstrated that the abnormalities in monocyte function can impair immune homeostasis, thereby activate autoimmune responses and result in SLE-associated immune-mediated damage ${ }^{5,6}$. Whereas, monocytes and consequent differentiation are verified as playing a decisive and crucial role in the systemic spread of HCMV as well as establishment of effective and persistent infection ${ }^{6}$. It is, therefore, possible that phenotypic 
and functional abnormalities caused by HCMV infection of monocytes play a crucial role in the occurrence and development of SLE. However, in the current literature, only a few studies have reported the effects of HCMV infection on monocyte function ${ }^{5,7}$.

HCMV encodes 252 open reading frames in the 230-kb genome and was believed to encode approximately 180 proteins ${ }^{8}$. HCMV infection involves significant changes in gene expression in different cell types at different stages of infection. However, a global investigation of differentially viral gene expression in HCMV-infected immune cells in SLE patients and their effects on cell function have never been performed. So, in this study, we utilized highthroughput RNA-Seq in combination with RT-PCR to explore the profile of HCMV gene expression in the peripheral blood mononuclear cells (PBMCs) of SLE patients. The effects and mechanism of SLE-associated US31 gene expression on monocyte immunological function were also investigated. The results of this study will provide a foundation for new concepts regarding SLE pathogenesis as well as novel strategies to prevent and treat this disease.

\section{Results}

\section{Global profile of HCMV gene expression in PBMCs of SLE} patients

To analyze the profile of HCMV gene expression in the PBMCs of SLE patients, we randomly selected PBMCs from three SLE patients for whole transcriptome sequencing and mRNA sequencing. Our data showed that 18 viral genes (UL29, UL32, UL34, UL36, UL37, UL44, UL50, UL56, UL82, UL84, UL95, UL105, UL112, UL117, UL123, US3, US31, and TRS1) were detected in the PBMCs of at least one of the three SLE patients. Of these, seven (UL34, UL44, UL82, UL84, UL95, UL112, and TRS1) were detected by both sequencing methods. Alternatively, UL36, UL123, and US31 were only detected during whole transcriptome sequencing, whereas UL29, UL32, UL37, UL50, UL56, UL105, UL117, and US3 were only detected during mRNA sequencing, respectively. Among 18 HCMV genes, seven (UL34, UL44, UL50, UL82, UL84, UL95, and UL11) were detected in the PBMCs of all three SLE patients, with four additional genes (US31, UL32, UL105, and TRS1) being detected from two SLE patients and seven (UL29, UL36, UL37, UL56, UL117, UL123, and US3) from one SLE patients. Moreover, 17 viral genes (UL29, UL32, UL34, UL37, UL44, UL50, UL56, UL82, UL84, UL95, UL105, UL112, UL117, UL123, US31, TRS1, US3) were also detected in the PBMCs of at least one of the three healthy controls whereasUL69, and IRS1 were only detected in healthy control PBMCs (Fig. 1a and Figure S1A). Cluster analysis indicated that UL44, UL82, UL84, UL95, and US31 had the highest expression levels (Fig. 1a and Figure S1A). PCR was also performed for 11 HCMV genes (UL32,
UL36, UL44, UL50, UL56, UL82, UL84, UL95, UL105, UL117, and US31) using normalized RNA quantities from another four SLE PBMC samples, further verifying their expression (Table S1, Fig. 1b).

\section{US31 is an SLE-associated HCMV gene}

Of the three HCMV genes detected only in the PBMCs of SLE patients during whole transcriptome but not by mRNA sequencing, UL36 and UL123 were confirmed as protein coding. US31 belongs to the US1 family and is highly expressed in M1 cells, M2 cells, and dendritic cells (DCs) derived from monocytes in HCMV-infected patients ${ }^{9}$. However, its function remains largely unknown ${ }^{8}$. In the present study, US31 gene expression was similarly detected in THP-1 cells infected with clinical HCMV strains (Figure S1B). Moreover, we also used RT-PCR to evaluate the expression of US31 in PBMCs from 73 SLE patients and 75 healthy controls. There was no significantly different in the positive rate for US31 detection in SLE patients (71.23\% [52/73]) comparing to healthy controls (61.33\% [46/75]) (Fig. 1c). but the level of US31 expression was significantly higher in SLE patients than in healthy controls $(P<0.001)$ (Fig. 1d), which was similar the detection of anti-HCMV antibody IgG titer whereas IgM titer showed no difference (Figure S1C and S1D). In contrast, anti-US31 antibody was significantly lower in SLE patients than in healthy controls (Figure S1E). We further explored the relationship between US31 expression in PBMCs and patient clinical features. Red blood cell numbers $(P=0.043)$, complement C3 levels $(P=0.028)$, and complement $C 4$ levels $(P=0.022)$ were significantly lower in US31-positive than US31-negative SLE patients (Table S2). The numbers of white blood cells, neutrophils, lymphocytes, and monocytes, 24-h proteinuria levels, Systemic Lupus Erythematosus Disease Activity Index (SLEDAI) score, anti-ds-DNA antibodies, U1-nuclear ribonucleoprotein (anti-U1RNP) antibodies, anti-histone antibodies, anti-Ro (anti-SS-A) antibodies, anti-La (anti-SS-B) antibodies, and other clinical indicators showed no significantly different in the US31-positive and US31-nagtive SLE patients.

\section{US31 is a highly conserved, protein-coding viral gene}

US31 sequence exhibits a high conservation in HCMV clinical isolates and the experimental strains. The homology of US31 in protein sequences is $99-100 \%$ in different human CMV strains, whereas its homology with viral genes from the other genus is 33-48\%. Additionally, homology analysis showed that the US31 gene and the US32 and US31 genes from Mandrillus leucophaeus cytomegalovirus, cynomolgus macaque cytomegalovirus strain Ottawa, and cercopithecine herpesvirus 5 all showed high levels of homology (Fig. 2a). To verify that US31 is protein coding, we cloned the US31 coding 


\section{A}

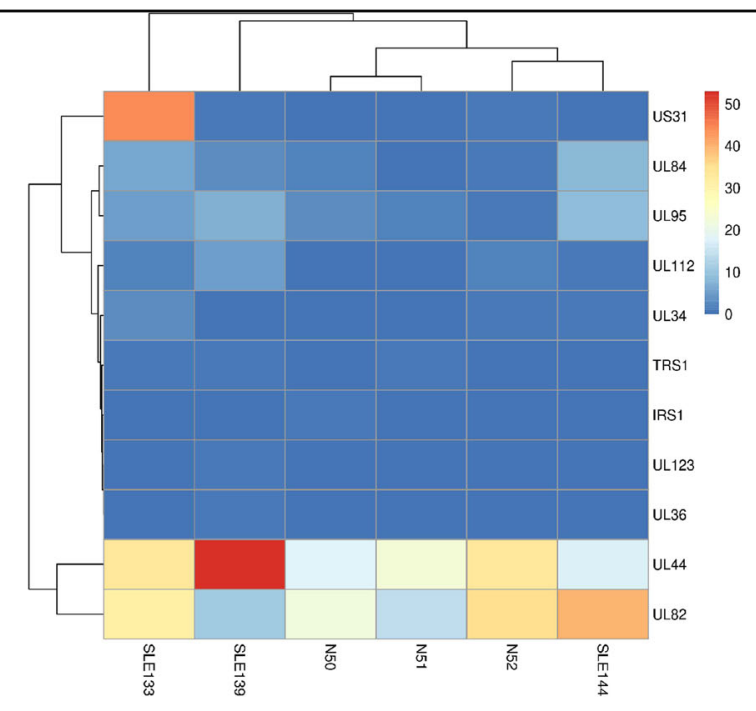

B
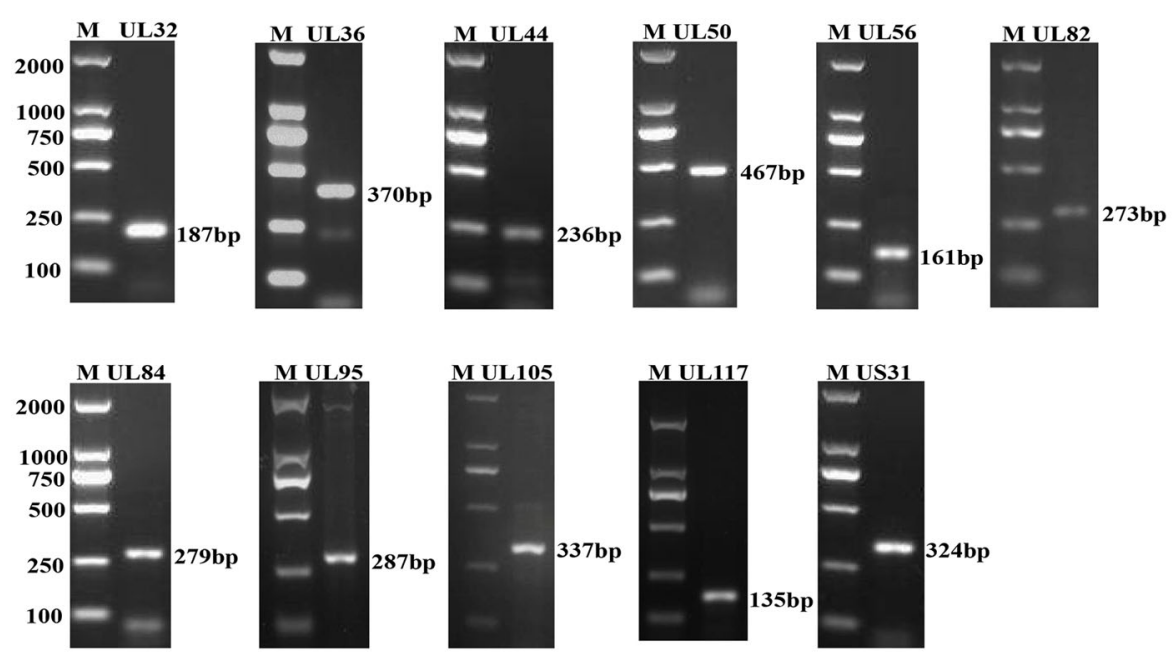

C
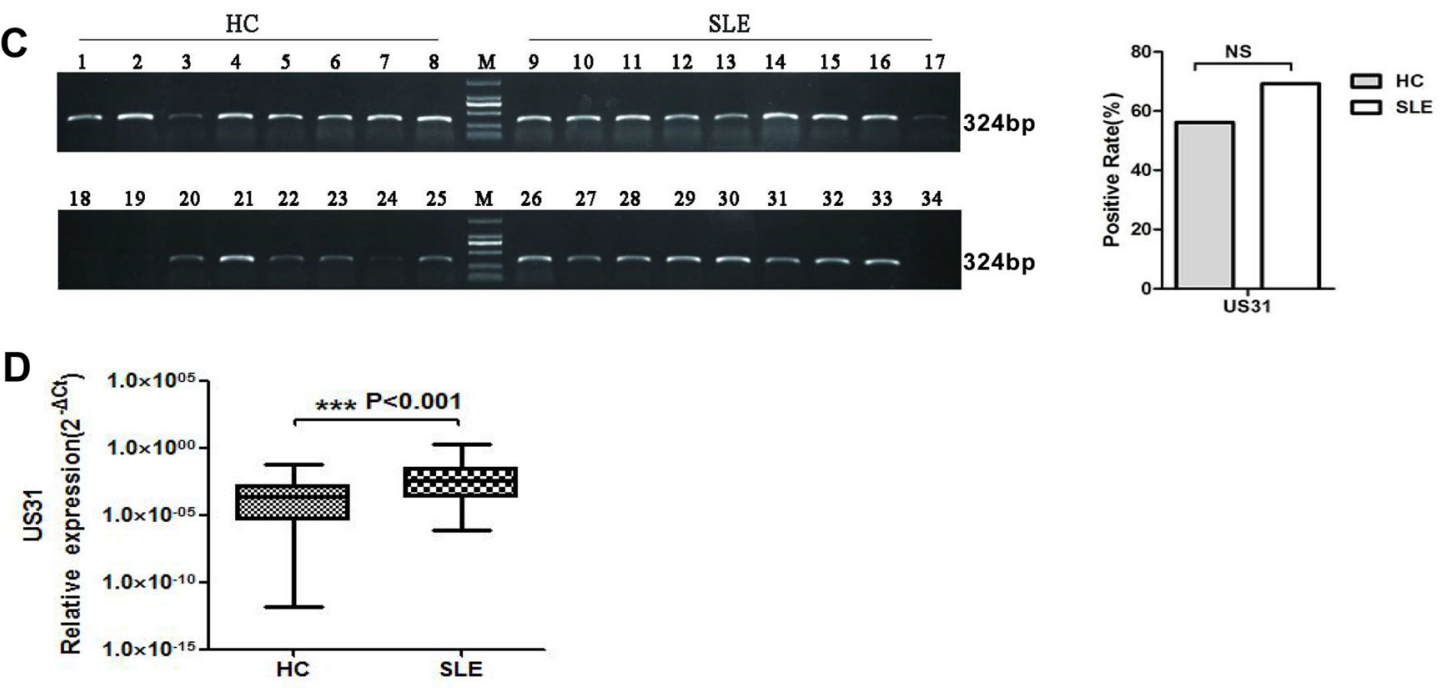

Fig. 1 (See legend on next page.) 


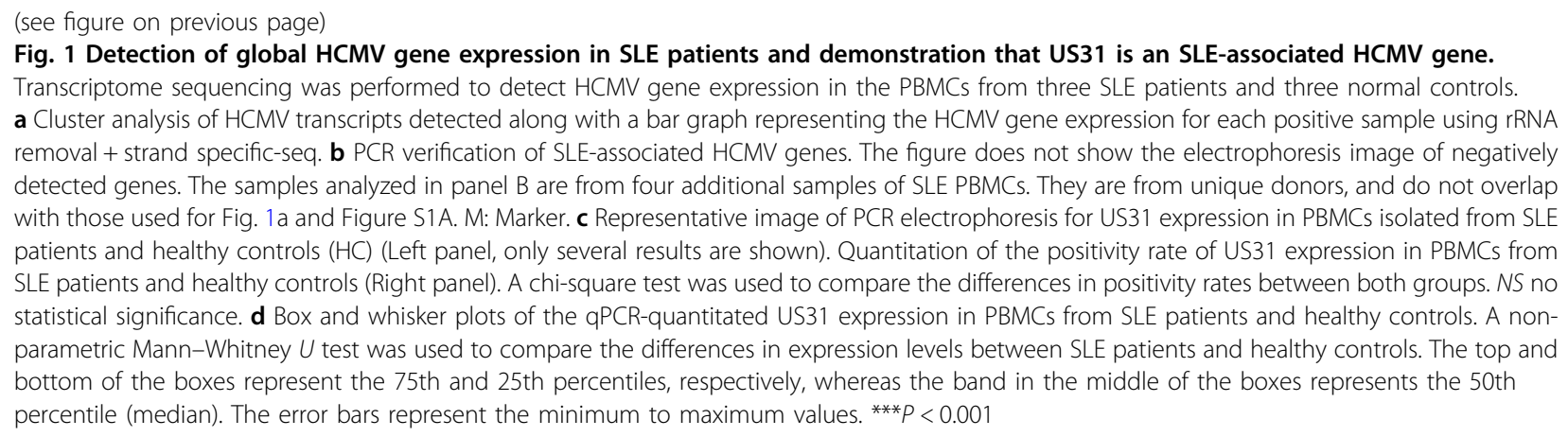

region, C-terminally tagged with $3 \times$ Flag tag, into an adenoviral vector (Ad-US31) or pcDNA3.1/US31 vector to infect or transfect HEK293T and COS-7 cells. We used an antibody against the $3 \times$ Flag tag as well as a self-made US31-specific antibody to detect US31 expression. We found that US31 protein is expressed in Ad-US31transfected cells (Fig. 2b) and appears to be synthesized as three species with molecular masses of 22, 24, and 26 $\mathrm{kDa}$ (predicted size is $20 \mathrm{kDa}$ ) in these cell types (Fig. 2c). Moreover, immunofluorescence microscopy also showed US31 protein expression (Fig. 2d). US31 sequence analysis showed that it contains multiple modification sites (Fig. 2e), which maybe the reason with higher molecular masses compared the predicted size. Structural analysis of the US31 gene suggests that US31 might constitute an E3 ubiquitin-protein ligase (ubr1), two zinc ion domain (Fig. 2f) and two possible nuclear localization signals (Fig. 2f, g), which indicated that US31 may participate in target protein degradation and highlight potential transcription factor function.

\section{US31 induces inflammation and stimulates THP-1/THP- 1-derived macrophage differentiation toward an M1 macrophage phenotype}

Transcriptome sequencing was used to explore the effects of US31 expression on the function of THP-1 monocytes and THP-1-derived macrophages. There were 133 differentially expressed genes in US31-expressed THP1 cell (Table S3) and 92 differentially expressed genes in THP-1-derived macrophages (Table S4) comparing to control groups. A subset of 18 randomly genes were chosen to verify the RNA-Seq data and showed highly concordant $(r=0.63$, Pearson correlation) (Figure S2A, Fig. S2b, c). A similar qRT-PCR analysis was used to confirm the quality and robustness of the RNA-Seq data of the transfected THP-1 cells (Figure S2D, Figure S2E). The consequent bioinformatics analysis indicated that US31 expression induces the inflammatory response in both THP-1 cells (GO:0006954, Table S5) and THP-1-derived macrophages (GO:0006954; Table S6). Especially, several hallmark changes were found in known NF-kB regulators and signaling molecules including an upregulation of interleukin (IL)-1 1 , RelB, TNF, ICAM1, IL-8, and CCL2 in US31expressed THP-1 cells and THP-1-derived macrophages (Table S7). Disease (by Biomarkers) analysis of the differentially expressed genes in both THP-1 cells and THP-1derived macrophages after transfection showed that US31 expression is related to the development of autoimmune diseases such as SLE (Figs. 3a, b).

Chan et al. ${ }^{7}$ showed that HCMV infection reprograms monocyte differentiation toward the M1 macrophage phenotype. Subsequently, Mukherjee et al. ${ }^{10}$ also found that non-classical monocytes from SLE patients display inflammatory features, further supporting the M1 phenotype. To further investigate the effects of Ad-US31 infection on THP-1/THP-1-derived macrophage differentiation, we compared the Ad-US31-infected cell transcriptomes to the known immunophenotypic profiles of M1 and M2 phenotype described by Martinez et al. ${ }^{11}$. Supplementary Tables S8-S11 show the lists of genes found to be strictly associated with M1 or M2 monocyte/ macrophage polarization. Compared to controls, 24 (51\%) and 23 (52\%) M1-associated genes were upregulated in US31-expressed THP-1 cells and THP-1-derived macrophages, respectively (Table S8 and S10). And a total of 16 of them are simultaneously upregulated in THP1 and THP-1-derived macrophages following infection with AdUS31 (Fig. 3c). In contrast, of the genes typically associated with the anti-inflammatory M2 phenotype, only 10 (28\%) and 9 (27\%) were upregulated in US31-expressed THP-1 cells and THP-1-derived macrophages, respectively (Table S9 and S11). These data show a clear transcriptional preference toward an M1 macrophage activation phenotype following Ad-US31 infection in both THP-1 cells and THP-1-derived macrophages.

\section{NF-KB2 is responsible for the immune function of US31 in mono-macrophages}

To identify the molecular mechanism of US31 during inflammation, we screened potential US31-interacting 
A

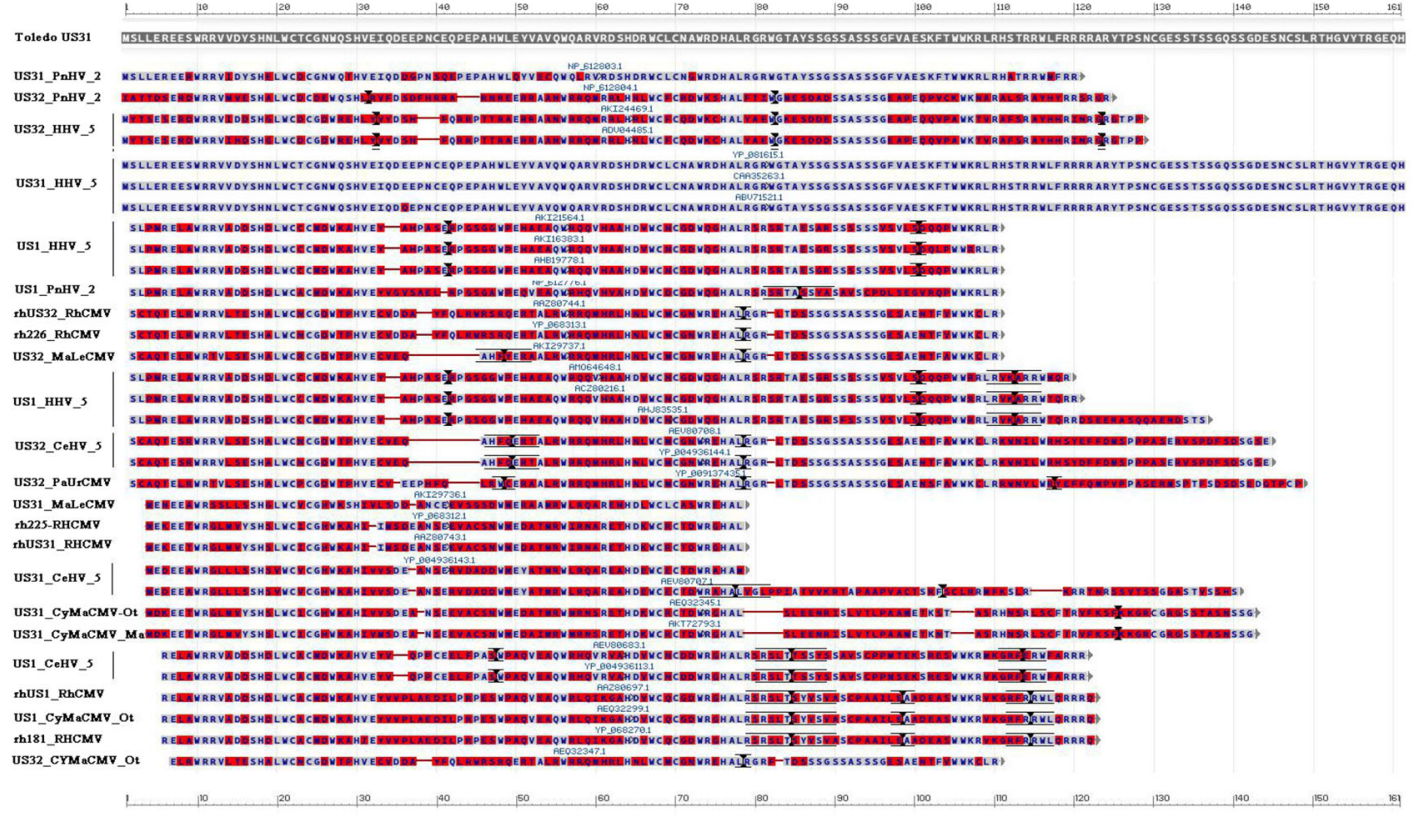

B

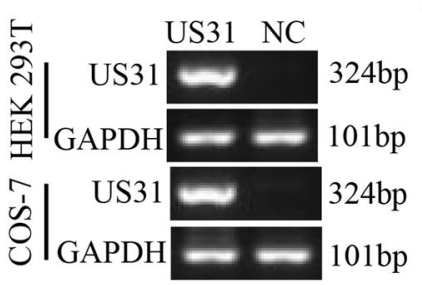

C

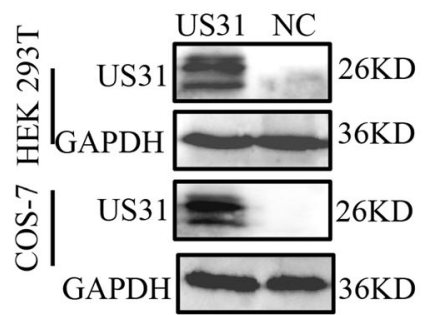

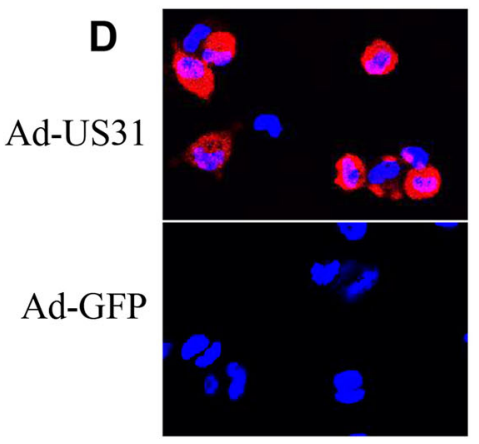

F
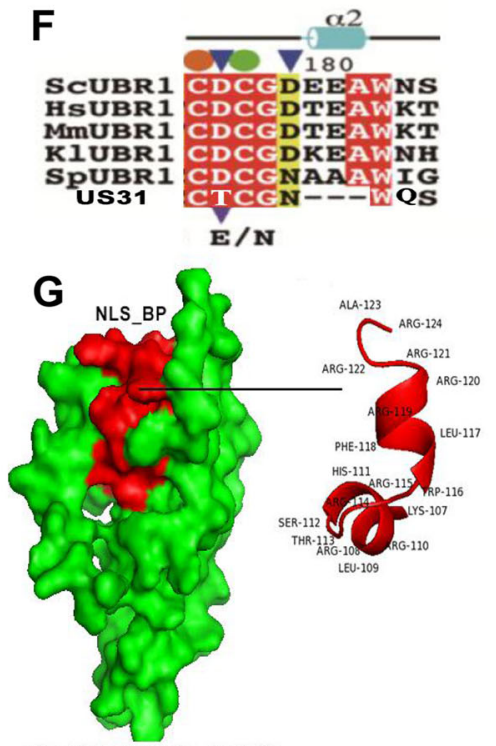

The third structure of pUS 31

Fig. 2 (See legend on next page.) 
Fig. 2 US31 gene expression. a NCBI Blast homology analysis of the US31 gene using the Toledo strain as a reference sequence. Blue represents identity with the reference strain whereas red represents differences. PnHV_2: Panine herpesvirus 2; HHV_5: Human herpesvirus 5; Rh_CMV: Rhesus Cytomegalovirus; MaLe_CMV: Mandrillus leucophaeus cytomegalovirus; CeHV_5: Cercopithecine herpesvirus 5; PaUrCMV: Papio ursinus

cytomegalovirus; CeHV: Cercopithecine herpesvirus 5; CyMaCMV_ot/ma: Cynomolgus macaque cytomegalovirus strain Ottawa/Mauritius. b mRNA detection of the US31 gene in HEK293T and COS-7 cells after infection/ transfection with Ad-US31 or Ad-GFP (NC, control) or pcDNA3.1/US31 vector (Figures do not show) for $24 \mathrm{~h}$. Expression was normalized to that of GAPDH. c Western blot analysis of US31 protein expression in HEK293T and COS7 cells $24 \mathrm{~h}$ after infection/ transfection with Ad-US31 or Ad-GFP (NC) or pcDNA3.1/US31 (Figures do not show). $d$ Analysis of US31 expression in AdUS31- or Ad-GFP (control)-infected THP-1-derived macrophages (48 h). Red staining indicates US31 expression (performed using a rabbit antibody against US31); blue staining indicates nuclei (DAPI). e Output of Expasy functional analysis of US31. f Sequence alignment of the UBR domain in Ubr1 E3 ligases from different organisms (Sc, Saccharomyces cerevisiae; Hs, Homo sapiens; Mm, Mus musculus; Kl, Kluyveromyces lactis; Sp,

Schizosaccharomyces pombe). Shading indicates residues that are identical (red) or highly conserved (yellow) in all sequences. Filled circles indicate residues that coordinate the zinc ions (orange for Zn1, green for Zn2). Key determinants for binding of the N-terminal residue of the substrate are marked with blue triangles and critical residues identified by previous genetic screening are indicated with purple triangles below the sequence ${ }^{39}$. g Predicted 3-dimensional protein structure of US31. The red area represents the nuclear localization signal sequence (see figure on previous page)

proteins using a customized protein binding array. 143 unique US31-interacting candidates were found in this screening assay (Table S12 and S13 Figure S3). Among these US31-interacting proteins, five proteins (NF-kB2, HSPA6, HSPA2, RNHI, and TXLNB) shown to be involved in immune system processes, and were chosen for further analysis. Notably, the functional groups assigned for this US31-interacting protein subset were largely related to NF-кB2 protein signaling (Fig. 4a). Co-immunoprecipitation (Co-IP) validated the direct interaction between US31 and NF-kB2. Endogenously expressed NF-kB2 protein was complexed with US31 following IP with an anti-US31 antibody (Fig. 4b). No detectable NF-kB2 was observed using control IgG. Furthermore, reciprocal Co-IP using an anti-NF- $\mathrm{KB} 2$ antibody confirmed this interaction. NF- $\mathrm{kB} 2$ is a member of the NF- $\mathrm{kB}$ transcription factor family that is well-known for its expression in multiple cell types and the role as a primary inflammation and immune function modulator $^{12-14}$. To evaluate the effects of this US31 and NFкB2 on immune function of mono-macrophages, we silenced NF- $\kappa$ B2 expression using si-RNA when US31 was expressed (Figure S4). The results showed that the NF- $\kappa$ B2 regulators and signaling molecules TNF- $\alpha$, IL-8, CCL2, ICAM1, and RelB were significantly downregulated (Fig. 4c). These data directly indicated the change of cell function with US31expression was contributed the NF-kB2 activation in mono-macrophages.

\section{US31 contribute to the ubiquitination of NF-KB2 precursor and consequent activation}

It is well know that, during the NF- $\mathrm{kB} 2$ activation, the p100 of NF- $\mathrm{kB} 2$ precursor is targeted for proteasomal processing to $\mathrm{p} 52$ of activated NF- $\mathrm{\kappa B} 2$. Then the $\mathrm{p} 52 / \mathrm{RelB}$ dimer then translocates into the nucleus to activate gene transcription. Subcellular fraction analysis by western blot indicates nuclear upregulation of both p52 of activated NF- $\kappa$ B2 and RelB protein (Fig. 4d). These data further confirmed the NF- $\mathrm{kB}$ activation by US31 protein. It has been demonstrated that the phosphorylation, polyubiquitination, and proteasomal processing of precursor p100 are required for NF-KB2 activation ${ }^{15}$. In vivo p100 phosphorylation (S866/870) and ubiquitination assays showed that p100 phosphorylation and ubiquitinated p100 accumulate in the presence of MG132 proteasome inhibitor when US31 is expressed (Fig. 4e, f). These data indicated that US31 promotes the ubiquitination of the phosphorylated p100 and consequently proteasomal processing to p52 and NF- $\mathrm{kB}$ activation. As the structural analysis of the US31 amino acid sequence suggests that it could be an E3 ubiquitin-protein ligase (ubr1), it was essential to evaluate the potential role of this protein in the processing of NF$\kappa \mathrm{B} 2$ during immune regulation. In addition, immunofluorescence microscopy of Ad-US31-infected THP-1derived macrophages shows that US31 protein is primarily expressed in the cytoplasm and low amounts are present in the cell nucleus (Fig. 2d). As NF-KB2 protein has a similar expression pattern, with high levels in the cytoplasm and low levels in the cell nucleus ${ }^{16,17}$, it is possible that US31 could act as E3-like ligase and promote NF-kB2 activation.

\section{Discussion}

Prior analysis of monocytes at 4 days after infection showed HCMV induces monocyte cell death, promotes inflammatory responses, induces cell cycle blockage, and participates in SLE development ${ }^{5}$. Moreover, transcriptome sequencing of monocytes after $4 \mathrm{~h}$ of infection indicated that HCMV also reprograms monocyte differentiation toward M1 macrophages ${ }^{7}$. However, the current understanding about HCMV gene expressions in PBMCs of SLE patients is limited.

Simultaneous performance of two library preparation methods to analyze global HCMV gene expression in the PBMCs of SLE patients afforded a more complete, unbiased understanding of viral gene expression in these samples. Specifically, poly(A)-Seq detected a total of 16 


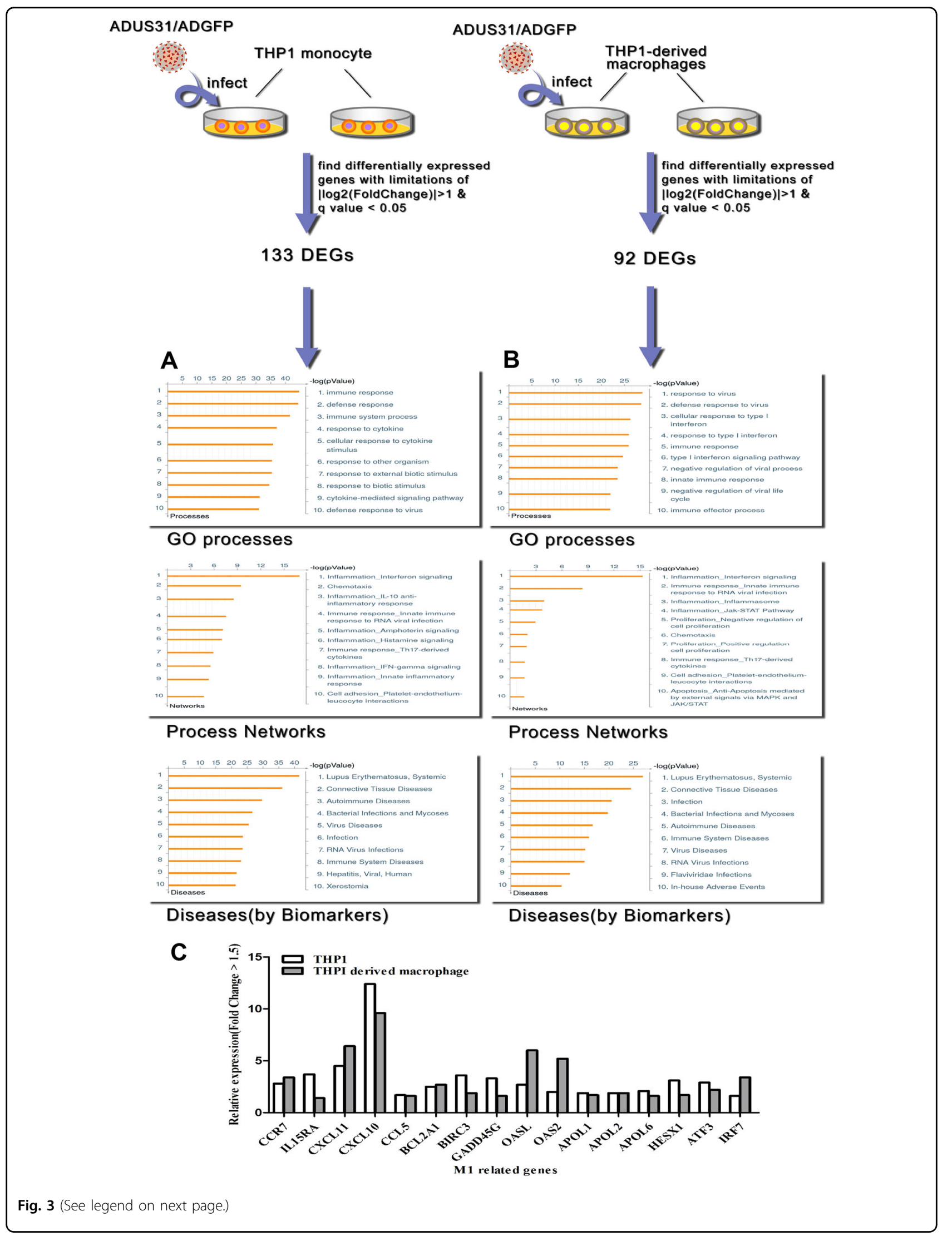


(see figure on previous page)

Fig. 3 Effect of US31 expression on cellular function of monocytes/macrophages. The most significant GO Process, Process Network, and Disease (by Biomarkers) terms for the differentially expressed genes detected in our RNA-Seq analysis of Ad-US31-infected THP-1 cells a and THP-1derived macrophages $\mathbf{b}$. The terms were filtered in accordance with $P<0.05$ and FDR $<0.05$. The top 10 significantly enriched terms are shown (software from Thomson Reuters Corporation was used to analyze differentially expressed genes). c A total of 16 of the M1-associated genes are upregulated in THP1 and THP-1-derived macrophages following infection with Ad-US31 (Fold change > 1.5 except IL15RA is 1.4 in THP-1-derived macrophage)

A

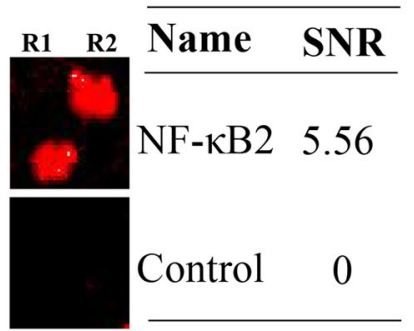

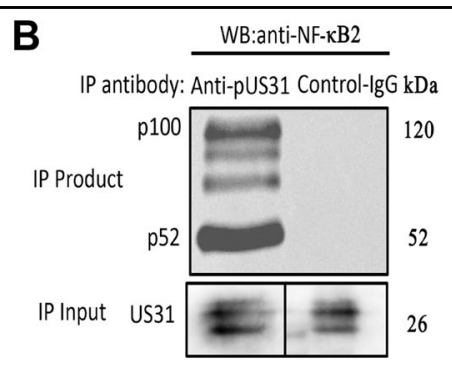

\section{Ad-US31 Ad-GFP}

IP antibody: Anti-NF-kB2 Anti-NF-kB2 kDa



$\mathrm{p}$

IP Input

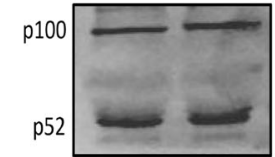

120

52
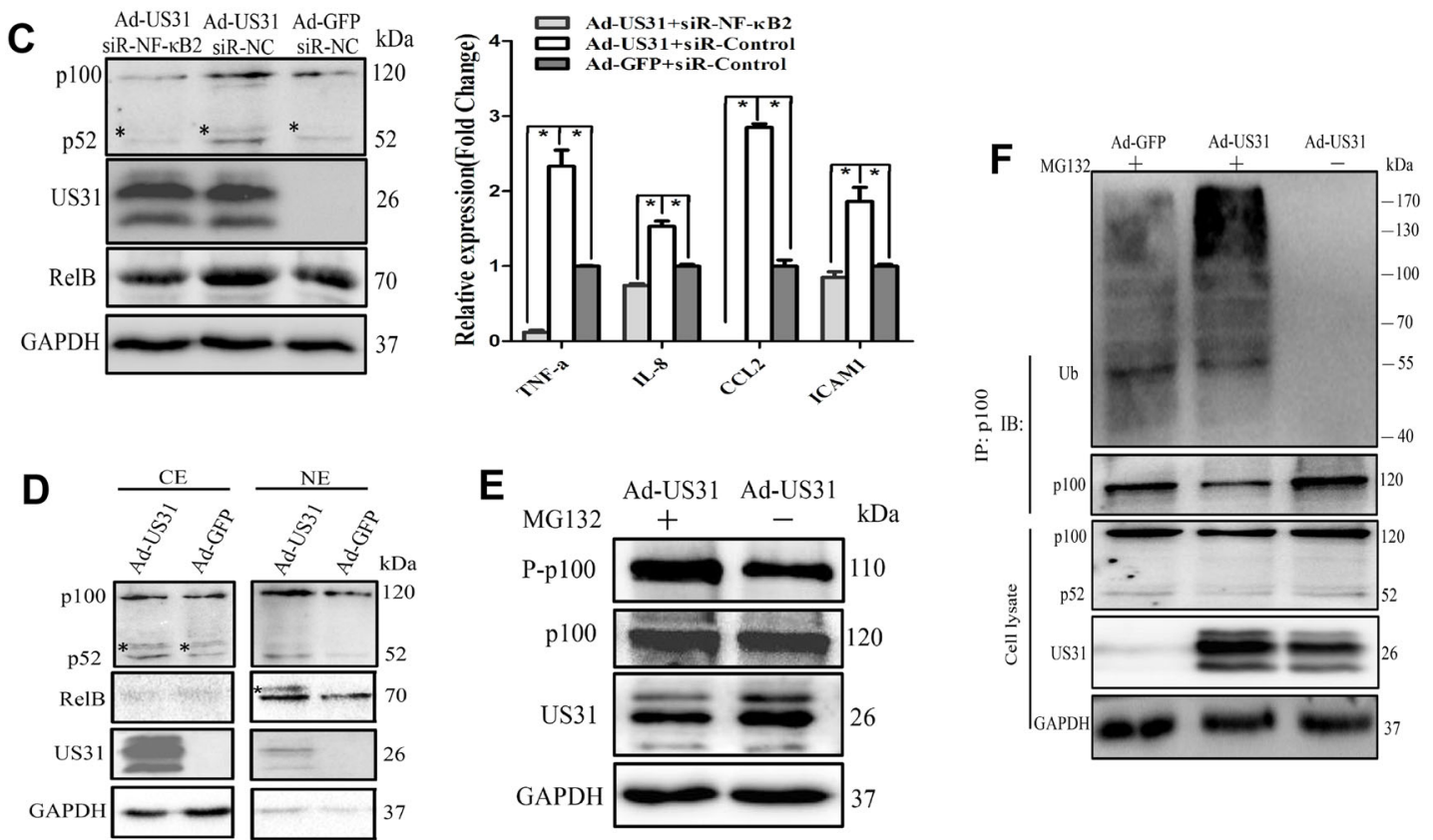

Fig. 4 US31 interacts with NF-KB protein signaling. a The interaction between US31 and NF-KB2 was screened by a human proteome microarray against pure US31 protein. The signal-to-noise ratio (SNR) indicated the average ratio of signal to noise calculated from two duplicate spots. R1: repetition 1; R2: repetition 1. b US31 forms a complex with NF-KB2 in Ad-US31-infected THP-1-derived macrophages (48 h) that can be detected following both US31/Flag and NF-KB2 immunoprecipitation (IP) and subsequent western blot analysis with the other antibody as shown (Figures do not show the IP results of anti-Flag). In each experiment, lysates incubated with control lgG (Antibody purified from control group immune serum) served as negative controls. The immunoprecipitation assays are representative of 3 independent experiments. c Inhibition of US31 in cell inflammation was verified by siRNA-NF-KB2. THP1 derived macrophages at $48 \mathrm{~h}$ post infection with Ad-US31 (or Ad-GFP) and NF-KB2 siRNA (or nonfunctional siRNA (siR-NC) by western blot and qRT-PCR. qRT-PCR results were presented as the means \pm SEM of three individual experiments in each group. *Non-specific band. d Western blot analysis of NF-KB2 (p100, p52) and RelB in subcellular fractions obtained from THP-1-derived macrophages. CE, cytoplasmic extract; NE, nuclear extract. *Non-specific band. e Phosphorylation of p100 in vivo. About 48 h postinfection, THP1 derived macrophages were incubated with a proteasome inhibitor, MG132 $(30 \mathrm{mM})$, for $6 \mathrm{~h}$ and then lysed in RIPA buffer. The phosphorylation and total levels of p100 were monitored. $\mathbf{f}$ US31 promotes the in vivo ubiquitination of NF-KB2. THP1-derived macrophages at $48 \mathrm{~h}$ post infection with AdUS31 (or Ad-GFP) and MG132 by IP and western blot 
genes, whereas whole transcriptome sequencing detected 11 genes. Among these, 8 genes were detected in both sequencing methods.

Notably, the most of the detected viral genes in the PBMCs of SLE patients have been shown to be associated with HCMV latency ${ }^{8}$. For example, UL32, a major tegument protein that participates in virus assembly, maturation and release, gene expression and modulation, as well as host cell cycle modification and protein synthesis, is associated with latency-associated process ${ }^{18}$. Alternatively, UL36 participates in cell apoptosis, gene expression regulating, and encoding toxic proteins ${ }^{19}$. $\mathrm{UL}_{4} 4^{18,20}, \mathrm{UL}^{121,22}{ }^{21} \mathrm{UL}^{23}{ }^{23}$, UL82 ${ }^{24}$, UL84 ${ }^{21}, \mathrm{UL}^{21}{ }^{21}$, $\mathrm{UL} 105^{18}$, and $\mathrm{UL} 117^{25}$ also function in various latencyassociated processes. Therefore, HCMV is in a latent infection state in the PBMCs of SLE patients.

There are currently three published studies concerning HCMV gene expression in monocyte or CD34+ haematopoietic cells.. Goodrum et al. ${ }^{18}$ used an in vitro model of HCMV latent infection in CD34+ cells, followed by HCMV cDNA array and found 68 HCMV genes distinct from HCMV productive infections. Similarly, Cheung et al. $^{26}$ also used gene arrays to evaluate viral gene expression in latent HCMV-infected myeloid cells, and observed 37 differentially expressed genes. Lastly, highthroughput sequencing analysis of HCMV gene transcripts in experimental and natural HCMV CD14+ monocyte and CD34+ cell infection detected changes in a total of 20 genes $^{21}$. Compared to these lists of differentially expressed genes $(68,37$, and 20 genes, respectively), we detected only 7 (UL32, UL34, UL44, UL69, UL84, UL105, and UL123), 2 (UL56, UL123), and 6 (UL29, UL37, UL44, UL50, UL84, and UL95) of the same genes in our study, respectively (Table S14). The difference between the present and previously published findings may result from different detection methods, objects of study and patterns of experimental and natural HCMV infection. Furthermore, US31 expression, which our results indicate as a disease-related gene, was not investigated by the other studies.

However, the US31 gene identified during our analysis has not been investigated with regard to its expression mode and function. As expression of the proliferation-associated HCMV IE2 86 gene inhibits US31 expression, US31 may also be a HCMV latencyassociated gene ${ }^{27}$. Notably, although neither Goodrum et al. ${ }^{18}$ or Cheung et al. ${ }^{26}$ detected the expression of US31, our data indicate that the positive rate of US31 gene expression in SLE patients is indeed higher compared to that in healthy control group, as confirmed using qPCR. Furthermore, compared with US31negative SLE patients, the RBC numbers, complement C3 levels, and complement C4 levels in the US31-positive SLE patients were significantly lower, suggesting that US31 expression is involved in SLE development.

A study by Van Damme et al. ${ }^{9}$, the results of which we confirmed (Figure S5A), also shows high US31 expression in HCMV-infected monocyte-derived DCs, M1, and M2 cells. To explore the function of US31 in the monomacrophage, recombinant adenovirus encoding US31 protein were used to infect THP-1 cells and THP-1derived macrophages before transcriptome sequencing. Subsequent bioinformatic analysis showed that US31 mainly participates in the inflammatory response and a predominantly M1 macrophage phenotype. Using a $\mathrm{HuProt}^{\mathrm{TM}}$ human protein array combing with Co-IP experiments and reciprocal siRNA silencing test, we identified NF- $\mathrm{kB} 2$ is responsible for the immune function in mono-macrophages by direct interaction with US31.

$\mathrm{NF}-\mathrm{\kappa B}$ regulates a number of key genes involved in cellular processes such as proliferation, apoptosis, and inflammation ${ }^{28}$. Traditionally, activation of NF- $\mathrm{KB}$ signaling is mediated through either canonical or noncanonical signaling pathways ${ }^{15}$. Non-canonical NF- $\mathrm{kB}$ signaling involves activation of the RelB/p52 NF- $\mathrm{kB}$ complex using a mechanism that relies on the polyubiquitination and proteasomal processing of the phosphorylated p100. The processing of p100 serves to generate p52 as well as induce the nuclear translocation of the RelB/p52 heterodimer ${ }^{15}$. Indeed, we found that US31 overexpression not only promoted the conversion of p100 to p52 as well as the translocation of the RelB/p52 complex into the nucleus, but also upregulated RelB and p100 expression (Figure S5B and S5C). Phosphorylated and ubiquitinated p100 accumulate in the presence of proteasome inhibitors MG132 when US31 is expressed. The inflammatory markers, including ICAM1, CCL2, IL-8, and TNF, downregulated after siRNA of NF- $\mathrm{kB} 2$ was transfected. These results clearly indicate that HCMVinduced US31 expression likely alters the expression of inflammation-related genes diseases via direct interaction with factors involved in the non-canonical NF-KB2 pathway. As the US31structure contained the homological domain of E3 ubiquitin-protein ligase (ubr1), it is possible that US31 could act as E3-like ligase and promote NF-kB2 activation. Additional details of this mechanism require further in-depth study (Fig. 5) as well as confirmation using infection with HCMV viruses with deficient/nonfunctional US31.

In summary, we provided the first global investigation of HCMV genes expression in PBMCs from SLE patients and demonstrated that US31 expression could change the immunological function of mono-macrophages and promote the M1 inflammatory response. In addition, we clarified for the first time that US31 could act as E3-like ligase and promote the ubiquitination of the phosphorylated p100 and proteasomal processing to consequently 


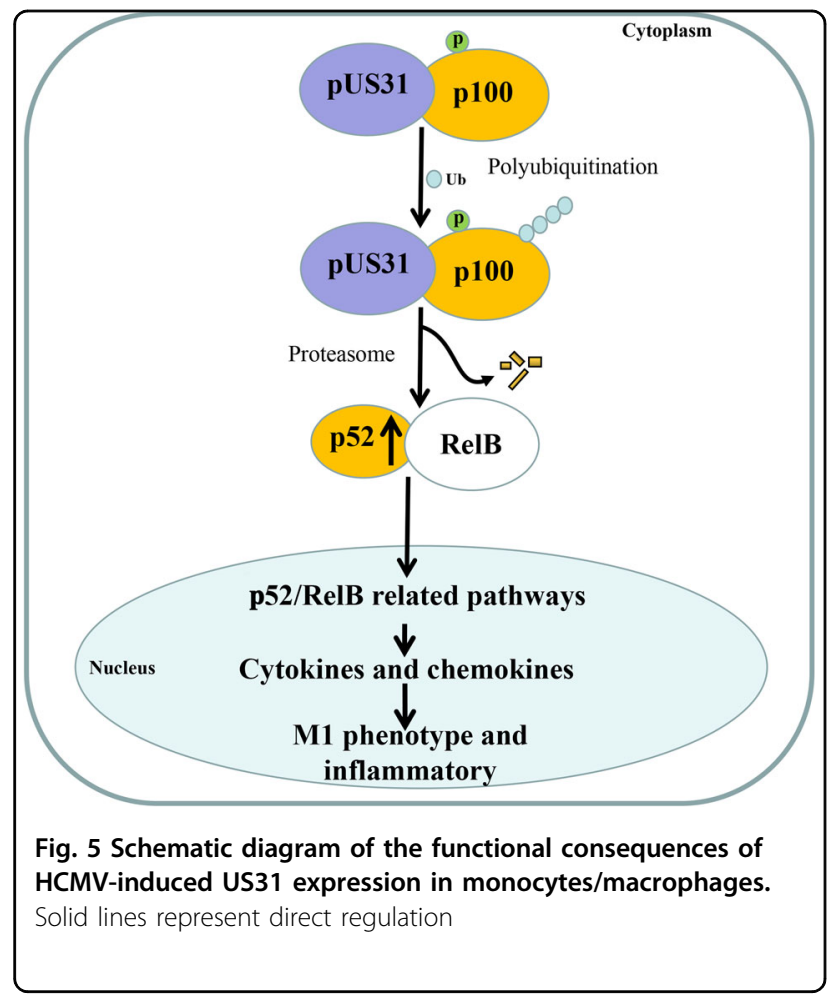

activate NF-KB2 pathway. The elucidation of this mechanism provides valuable insight for the future development of novel therapeutic options for SLE patients, with the ultimate goal of treating and/or preventing the development or HCMV-induced exacerbation of this prevalent autoimmune disease.

\section{Patients and methods \\ Study population}

A total of 87 SLE patients (73 women and 14 men), admitted to the Division of Rheumatology of the First and Second Affiliated Hospital of Wenzhou Medical University between October 2014 and May of 2016, were enrolled in this study. Written informed consent was obtained from the patients who participated in the study. The procedures of this study followed were in accordance with the ethical standards of the responsible committee on human experimentation (the hospital of the First and Second Affiliated Hospital of Wenzhou Medical University, China) and with the Helsinki Declaration of 1975, as revised in 2008. The Medical Ethical Committee of the hospital of the First and Second Affiliated Hospital of Wenzhou Medical University approved the consent procedure and subsequent testing protocols as well as anonymization and use of the data. All patients were in compliance with the classification standard set by the American College of Rheumatology in $1997^{29}$. The corresponding author, Xiangyang Xue, was responsible for anonymizing the data collected from participants and all of the data were used and analyzed in strictly anonymous form. Patients were aged from 21 to 77 years (mean 35.6 \pm 13.9 years). All SLE patients were tested using a common clinical symptom survey and common testing indices and scored in accordance with the internationally accepted SLEDAI ${ }^{30}$. Blood samples from 79 healthy subjects (62 women and 17 men; 20-59-years-old) formed the normal population control group. Individuals infected with hepatitis B, hepatitis C, HIV, syphilis, Epstein-Barr virus, or who had other infections were excluded from both groups.

\section{Extraction of RNA from PBMCs}

Blood (4 ml) was collected intravenously and placed in ethylenediamine tetra-acetic acid anticoagulant tubes. PBMCs were isolated by using human peripheral blood lymphocyte separation medium (Tianjin Hao Yang Biological Manufacture, Tianjin, China) according to the manufacturer's protocol. Following isolation, PBMCs were washed twice with phosphate-buffered saline (PBS), and total RNA was extracted by using TRIzol reagent (Invitrogen Life Technologies, Grand Island, NY, USA) according to the manufacturer's protocol. Potential DNA contamination in the purified RNA samples was avoided by using DNAfree reagent (Ambion, Carlsbad, CA, USA) dissolved in diethyl pyrocarbonate-treated water. RNA samples were stored at $-80^{\circ} \mathrm{C}$ after spectrophotometerbased measurement of the concentration.

\section{Deep sequencing analysis of $\mathrm{HCMV}$ gene expression in PBMCs}

The total RNA from the PBMCs of three SLE patients and three normal healthy controls (SLEDAI scores $>15$, all women) was extracted to analyze HCMV gene expression. The anti-HCMV IgM and IgG levels in these three SLE patients were $0.296 \pm 0.49$ COI (mean \pm standard error) and $269.867 \pm 67.38 \mathrm{U} / \mathrm{ml}$ (mean \pm standard error), respectively. Considering that the number of HCMV-infected PBMCs is low, and viral gene expression is low as well, we carried out mRNA (PolyA library) and whole transcriptome (strand-specific library) deep sequencing simultaneously in all six samples. The total RNA isolated from each sample was quantified and qualified using an Agilent 2100 Bioanalyzer (Agilent Technologies, Palo Alto, CA, USA), NanoDrop (Thermo Fisher Scientific Inc., Waltham, MA, USA), and $1 \%$ agarose gel electrophoresis. Total RNA $(1 \mu \mathrm{g})$ with a RIN value above 7 was used for library preparation.

Poly(A) mRNA isolation was performed using an NEBNext Poly(A) mRNA Magnetic Isolation Module (NEB, Ipswich, MA, USA), whereas mRNA fragmentation and priming was conducted using NEBNext First Strand Synthesis Reaction Buffer and NEBNext Random Primers. 
First strand cDNA was synthesized using ProtoScript II Reverse Transcriptase, and second-strand cDNA was synthesized using Second Strand Synthesis Enzyme Mix. The purified double-stranded cDNA (performed using AxyPrep Mag PCR Clean-up [Axygen, Union City, CA, USA]) was then treated with End Prep Enzyme Mix to repair both ends and add a dA-tail in one reaction, followed by a T-A ligation to add adaptors to both ends. Size selection of Adaptor-ligated DNA was then performed using AxyPrep Mag PCR Clean-up (Axygen), and fragments of approximately $360 \mathrm{bp}$ (with the approximate insert size of $300 \mathrm{bp}$ ) were recovered. Each sample was then amplified by PCR for 11 cycles using P5 and P7 primers, with both primers carrying sequences that would anneal to the flow cell for bridge PCR. The P7 primer also carried a six-base index that allows multiplexing. The PCR products were again cleaned up using AxyPrep Mag PCR Clean-up (Axygen), validated using an Agilent 2100 Bioanalyzer (Agilent Technologies), and quantified with a Qubit 2.0 Fluorometer (Invitrogen). Then, libraries with different indices were multiplexed and loaded onto an Illumina HiSeq instrument according to the manufacturer's instructions (Illumina, San Diego, CA, USA). Sequencing was carried out using a $2 \times 150$ bp paired-end (PE) configuration. Image analysis and base calling were conducted with HiSeq Control Software (HCS)+OLB +GAPipeline-1.6 (Illumina).

In order to perform whole transcriptome deep sequencing, the rRNA was first removed from the total RNA using a Ribo-Zero ${ }^{\mathrm{TM}}$ rRNA Removal Kit (Human/Mouse/Rat) (Illumina). The ribosomal-depleted RNA was then fragmented and reverse-transcribed. First strand cDNA was synthesized using ProtoScript II Reverse Transcriptase with random primers and Actinomycin D. Second-strand cDNA was synthesized using Second Strand Synthesis Enzyme Mix (including dACG-TP/dUTP). Subsequent steps were the same those listed above for mRNA deep sequencing.

In order to detect HCMV sequences in the sequencing reads, Cutadapt software was first used for pre-processing of the raw data, allowing the low-quality data to be filtered and the contaminated and adapter sequences to be removed (settings other than default parameters: -q 20, $20 ; \quad-\mathrm{m} \quad 75 ; \quad--\max -\mathrm{n}=0.1 ; \quad$-e 0.1$)$. Further, Bowtie2 software was used to align the clean data reads with the viral genome from the publicly available reference genomes for Merlin (GenBank accession number: NC_006273.2) and TB40E (GenBank accession number: EF999921) (settings other than default parameters: --verysensitive; --very-sensitive-local). Transcripts in fasta format were converted from known gff annotation file and indexed properly. Then, using the reference gene file, HTSeq (v0.6.1) was used to estimate gene expression levels from the pair-end clean data (no special parameters).

\section{PCR detection of HCMV genes in PBMCs}

In order to quantitate 18 of the HCMV genes detected during the RNA-Seq analysis, we first obtained 17 HCMV strains from the National Center for Biotechnology Information (NCBI) database. The gene sequences corresponding to each strain are as follows: HAN31 (GenBank: JX512208.1), U11 (GenBank: GU179290.1), HAN20 (GenBank: GQ396663.1), HAN13 (GenBank: GQ221973.1), HAN38 (GenBank: GQ396662.1), Merlin (GenBank: AY446894.2), JP (GenBank: GQ221975.1), JHC (GenBank: HQ380895.1), AD169 (GenBank: FJ527563.1), 3301 (GenBank: GQ466044.1), 3157 (GenBank: GQ221974.1), VR1814 (GenBank: GU179289.1), AF1 (GenBank: GU179291.1), TR (GenBank: KF021605.1), U8 (GenBank: GU179288.1), Toledo (GenBank: GU937742.2), and Towne (GenBank: FJ616285.1). Homology analysis was carried out, and Primer 6.0 software was used to design upstream and downstream primers in the conserved regions of every gene. The designed primers were then aligned with the NCBI Primer-BLAST $\mathrm{nr}$ database to exclude the amplification of host genes and genomes of other herpesviruses. For quantitation of HCMV gene expression in PBMCs, reverse transcription was carried out on RNA samples from PBMCs isolated from four SLE patients. First-strand cDNA was reverse transcribed using a ReverTraAce ${ }^{\circ}$ qPCR RT Kit (Toyobo $^{\circ}$, Tokyo, Japan) from $1 \mu \mathrm{g}$ of total RNA (denaturation at $65^{\circ} \mathrm{C}$ for $5 \mathrm{~min}$, incubation at $37^{\circ} \mathrm{C}$ for $15 \mathrm{~min}$, heat at $98^{\circ}$ $\mathrm{C}$ for $5 \mathrm{~min}$ ). The reaction solution (X $\mu \mathrm{l}$ nuclease-free water, $2 \mu \mathrm{l} 5 \times$ RT buffer, $0.5 \mu \mathrm{l}$ RT Enzyme Mix, $0.5 \mu \mathrm{l}$ Primer Mix, and $1 \mu \mathrm{g}$ RNA) was stored at $-20^{\circ} \mathrm{C}$. The PCR reaction conditions consisted of denaturation at $95^{\circ} \mathrm{C}$ for $5 \mathrm{~min}$, followed by 35 cycles of $95^{\circ} \mathrm{C}$ for $30 \mathrm{~s}$, annealing temperature (gene specific) for $30 \mathrm{~s}, 72^{\circ} \mathrm{C}$ for $30 \mathrm{~s}$, and a final extension at $72^{\circ} \mathrm{C}$ for $10 \mathrm{~min}$. The PCR reaction solution consists of template $(1 \mu \mathrm{g})$, primer $1(10$ $\mu \mathrm{M})$, primer $2(10 \mu \mathrm{M})$, Taq Polymerase $(0.1 \mathrm{U} / \mu \mathrm{l})$, and water up to $25 \mu$ l (TIANGEN Biotech C., Ltd., Beijing, China). The amplified products were electrophoresed on a $1.5 \%$ agarose gel containing ethidium bromide $(\mathrm{EB})$ and the bands were photographed. Primers and reaction conditions are listed in Table S1.

\section{Quantitation of US31 in THP-1 cells infected with clinical viral strains}

THP-1 cells (American Type Culture Collection (ATCC ${ }^{\circ}$ ), Manassas, VA, USA) were cultured using RPMI 1640 medium supplemented with $10 \%$ fetal bovine serum (FBS). HCMV clinical strains 3-2 were preserved by the Department of Microbiology and Immunology, Wenzhou Medical University. In order to detect US31 expression in HCMV-infected monocytes, THP-1 cells were infected with $\mathrm{HCMV} 3-2(\mathrm{MOI}=5)$ for $3 \mathrm{~h}$ at $37^{\circ} \mathrm{C}$. The cells were cultured in fresh medium with $10 \%$ FBS continuously for 
15 days. Cells were collected every day for RNA extraction and RT-PCR was carried out as described above to quantitate US31 expression.

\section{Testing of HCMV-specific serum IgG and IgM and anti- US31 serum level}

HCMV-specific IgG and IgM antibodies in serum samples from SLE patients and normal controls were detected by enzyme-linked immunosorbent (ELISA) using an anti-CMV IgG and IgM test kit according to the instructions of the manufacturer (Roche, Mannheim, Germany). The ELISA tests were performed using a fully automated ELISA processor (Roche). Recombinant US31 purified from BL21/pET21a-US31 was used as antigen to detect the anti-US31 serum level in SLE patients and normal controls by ELISA.

\section{Preparation of recombinant US31 adenoviral overexpression vector}

Upstream and downstream primers were designed according to the US31 gene sequence (GenBank accession number: GU937742). The upstream primer sequence was 5'-TCCA-

\section{GACCCGGGACCGAATTCGCCACCATGTCGCTCTT}

GGAGCG-3', and the downstream primer sequence was 5'-CGCTCGAGATCTGTAGTGTTGTTCACCCCGTG

$\mathrm{T}-3^{\prime}$, with the underlined sequences containing an EcoRI restriction site. The PCR amplification conditions for the US31 gene were as follows: pre-denaturation at $95^{\circ} \mathrm{C}$ for 5 min followed by 25 cycles of denaturation at $94^{\circ} \mathrm{C}$ for $20 \mathrm{~s}$, annealing at $55^{\circ} \mathrm{C}$ for $20 \mathrm{~s}$, and extension at $72{ }^{\circ} \mathrm{C}$ for $30 \mathrm{~s}$, before a final extension of $72^{\circ} \mathrm{C}$ for $10 \mathrm{~min}$. The PCR product was verified using $1 \%$ agarose gel electrophoresis. The target segment was extracted with an agarose gel DNA extraction kit (TIANGEN) according to the manufacturer's instructions. Ligation was carried out to obtain pHBAd-MCMV-GFP-US31 recombinant plasmids with $3 \times$ flag tag, which were then transformed into DH5 $\alpha$ competent cells and verified by restriction enzyme mapping and DNA sequencing (Huada, Shanghai, China).

HEK293T and COS-7 cells were obtained from the ATCC ${ }^{\ominus}$. HEK293T cells were subcultured into $60-\mathrm{mm}$ cell culture dishes. When the cells reached $70-80 \%$ confluency, Lipofiter ${ }^{\mathrm{TM}}$ transfection reagent was used to transfect the recombinant pHBAd-MCMV-GFP-US31 vector plasmid into the cells. The cell culture medium was changed $6 \mathrm{~h}$ after transfection. Cells were observed for virus production every day, and when cytopathy was observed in the majority of the cells and these cells were detached from the plates, the viruses were harvested and stored at $-80^{\circ} \mathrm{C}$. Then, HEK293T cells were subcultured onto 96-well plates at a density of $1 \times 10^{4}$ cells/well. After the cells were adhered to the plate, the culture medium was removed and $100 \mu \mathrm{l}$ of serially diluted viral supernatant $\left(10^{-6}-10^{-13}\right)$ was added to each well. A total of 10 wells were subcultured with the same dilution as well as untreated negative controls. The cells were incubated at $37^{\circ} \mathrm{C}, 5 \% \mathrm{CO}_{2}$ for 10 days. The condition of the cells was observed on days 3 to 10 . On day 10, the cytopathogenic effect (CPE) status of every well was observed under a microscope and compared with the negative controls. The number of positive wells for samples in each row was recorded. The recombinant adenovirus titer $(\mathrm{d}=$ $\log _{10}$, dilution $=1, \mathrm{~s}=$ sum of positivity ratios) was calculated based on the virus activity formula $\mathrm{T}=10^{1+\mathrm{d}(\mathrm{s}}$ -0.5). Viral supernatants were sent to Hanbio Ltd. (Shanghai, China) for enrichment and purification. After purification, viral titers were $10^{10} \mathrm{PFU} / \mathrm{ml}$ and used for subsequent cell infection experiments. The Phyre2 Database (http://www.sbg.bio.ic.ac.uk/phyre2/html/page.cgi? id=index) was used for US31 amino acid structural analysis, whereas GalaxyWEB (http://galaxy.Seoklab.org/) was used to construct 3-dimensional models of US31.

\section{Induction of THP-1-derived macrophages and infection with US31 recombinant adenovirus}

The induction protocol for THP-1 derived macrophages has been previously described ${ }^{31}$. Briefly, $3 \times 10^{5}$ THP- 1 cells/well were subcultured in 6-well plates, and PMA (300 ng/2 ml; Sigma-Aldrich, St. Louis, MO, USA) was added and incubated for $24 \mathrm{~h}$ to induce differentiation. When the proportion of cells adhering to the walls of the plate was $70-80 \%$, PBS was used to wash the cells thrice to remove unsuccessfully induced cells floating in the culture medium. The remaining cells were THP-1-derived macrophages. Our previously reported multiplicity of infection (MOI) value for UL138 adenoviral transfection of monocytes was used as a reference ${ }^{32}$. In the present study, an MOI of 100, 500, or 1 for recombinant adenovirus ADU31 was used to infect THP-1 cells, THP-1-derived macrophages, or COS-7 and HEK293T cells, respectively. RT-qPCR and western blotting were used to quantitate US31 expression.

\section{US31 and inflammatory markers quantitation by} quantitative real-time reverse transcription PCR (RT-qPCR)

In order to analyze US31 gene expression levels in PBMCs from patients with SLE and healthy controls, $1 \mu \mathrm{g}$ of total PBMC-derived RNA was reverse transcribed with a ReverTraAce ${ }^{\circ}$ PCR RT Kit (Toyobo ${ }^{\circ}$, Tokyo, Japan). US31 expression was assessed by real-time PCR with $\mathrm{iQ}^{\mathrm{TM}}$ $\mathrm{SYBR}^{\circ}$ Green Supermix (Bio-Rad, Berkeley, CA, USA) and specific primers (forward, 5'-GTATTCCTCGGGTT CCTC-3'; and reverse, 5'-TTTCCCCACAGTTAGACG3'; $143 \mathrm{bp}$ ). Human $G A P D H$ was used as an internal control (forward, 5'-AACTCTGGTAAAGTGGATAT TG-3'; and reverse, 5'-GGTGGAATCATATTGGAACA$\left.3^{\prime} ; 87 \mathrm{bp}\right)$. The PCR program was $95^{\circ} \mathrm{C}$ for $3 \mathrm{~min}$, followed 
by 40 cycles of $95^{\circ} \mathrm{C}$ for $10 \mathrm{~s}, 55^{\circ} \mathrm{C}$ for $30 \mathrm{~s}$, and $72^{\circ} \mathrm{C}$ for $30 \mathrm{~s}$. The RT-qPCR reaction solution contains $100 \mathrm{ng}$ template, forward primer $(10 \mu \mathrm{M})$, reverse primer $(10$ $\mu \mathrm{M}), 2 \times \mathrm{iQ}^{\mathrm{TM}} \mathrm{SYBR}^{\circ}$ Green Supermix, and nuclease-free water up to $10 \mu \mathrm{l}$. qPCR was performed using a CFX96 Touch ${ }^{\mathrm{TM}}$ Real-Time PCR Detection System (BioRad). Melting curves were generated for each real-time PCR to verify the specificity of each PCR reaction. Duplication was performed for accuracy judgment. The comparative viral copy $\left(\mathrm{C}_{\text {virus }}\right)$ was normalized to the amount of GAPDH using the $2^{-\Delta \mathrm{Ct}}$ method, with $\triangle \mathrm{CT}=\mathrm{CT}_{\mathrm{UL31}}-$ $\mathrm{CT}_{\mathrm{GAPDH}}$. ICAM1, CCL2, IL-8, and TNF specific primers and program were previously reported ${ }^{33-36}$.

\section{Immunoblotting}

Total protein was extracted from transfected HEK293T, COS-7, THP1, and macrophage cells using protein lysis buffer (Beyotime Institute of Biotechnology, Beijing, China) supplemented with protease inhibitor cocktail (Pierce, Rockford, IL, USA) at $4{ }^{\circ} \mathrm{C}$ for $20 \mathrm{~min}$. Protein concentration was determined using a BCA assay kit. Protein samples $(20 \mu \mathrm{g} / \mathrm{lane})$ were separated using 10-12\% sodium dodecyl sulfate (SDS)-polyacrylamide gel electrophoresis and then electrophoretically transferred to polyvinylidene difluoride membranes (Millipore, Billerica, MA, USA). After blocking with $5 \%$ skim milk for $1.5 \mathrm{~h}$ at $37^{\circ} \mathrm{C}$, the membranes were incubated with primary antibody at $4{ }^{\circ} \mathrm{C}$ overnight. Antibodies against NF- $\mathrm{\kappa B} 2$ (p52/ p100), phospho- NF-kB2 p100 (Ser 866/870), ubiquitin (P4D1), RelB (Cell Signaling Technology, Beverly, MA, USA), and GAPDH (GOOD HERE, Hangzhou, China) were diluted with Primary Antibody Dilution Buffer (Beyotime Institute of Biotechnology) at a 1:1000 dilution, whereas the US31 antibody was diluted 1:5000 (this antibody was a self-prepared rabbit anti-US31 antibody from our laboratory). The membranes were then washed with TBST buffer five times for $5 \mathrm{~min}$ each and incubated with secondary antibody (horseradish peroxidase (HRP)conjugated goat anti-rabbit IgG (MULTI SCIENCES, Hangzhou, China)) for $1.5 \mathrm{~h}$ at $37^{\circ} \mathrm{C}$. The bands were detected using enhanced chemiluminescence and visualized with a Gel Doc 2000 (BioRad).

\section{Immunofluorescent localization of US31 protein}

Co-localization of cellular proteins in the transfected cells was observed by immunofluorescence. Briefly, THP1-derived macrophage $\left(2 \times 10^{5}\right.$ cells/well in 6 -well plates containing glass coverslips) were mock transfected or transfected with recombinant adenovirus encoding US31 for $48 \mathrm{~h}$. Macrophage cells were then fixed in $4 \%$ paraformaldehyde at $37^{\circ} \mathrm{C}$ for $10 \mathrm{~min}$ and permeabilized by $0.3 \%$ Triton X-100 at $37^{\circ} \mathrm{C}$ for $10 \mathrm{~min}$. After blocking the cells in PBS supplemented with $10 \%$ goat serum (Beyotime Institute of Biotechnology) for $60 \mathrm{~min}$ at $37^{\circ} \mathrm{C}$, the macrophages were stained with a rabbit antibody specific to US31. Secondary DyLight549-conjugated goat antirabbit antibody (MULTI SCIENCES) was applied in PBS supplemented with $10 \%$ goat serum for $1 \mathrm{~h}$ at $37^{\circ} \mathrm{C}$. Cells were subsequently incubated in Hoechst 33258 (Beyotime Institute of Biotechnology) for $5 \mathrm{~min}$ at room temperature. The coverslips were mounted on slides and visualized using a fluorescence microscope (Nikon C1-i, Tokyo, Japan).

\section{Effects of US31 expression on gene expression in THP-1 monocytes and THP-1-derived macrophages}

Total RNA was extracted with TRIzol from THP-1 and THP-1-derived macrophages after transfection with adenovirus US31 or control adenovirus. The RNA was then purified with magnetic Agencourt Ampure beads (APN 000132, Beckman Coulter, Brea, CA, USA), and DNAfree reagent (Ambion, Carlsbad, CA, USA) was added to remove DNA contamination. Total RNA was quantified using a NanoDrop ND-1000, and RNA integrity was assessed by standard denaturing agarose gel electrophoresis. A poly(A) library preparation was used (after library preparation was completed, a Qubit ${ }^{\circ}$ 2.0 Fluorometer was used for initial quantitation and to dilute the library to 1.5 $\mathrm{ng} / \mu \mathrm{l})$. Subsequently, an Agilent 2100 bioanalyzer was used to detect the insert size of the library, followed by qRT-PCR to quantitate the concentrations of the library and ensure quality $(>2 \mathrm{nM})$. Paired-end sequencing was carried out based on the Illumina HiSeq/MiSeq technology platform. In order to eliminate biological variation, thresholds were set for the fold difference and significance level of the differentially expressed genes (I $\log 2$ (FoldChange) $\mid>1$ and $q$-value $<0.005)$. In addition, for genes showing statistical differences, the Annotation, Visualization and Integrated Discovery (DAVID) system was used to analyze the main biological processes and pathways. GO analysis was also used to carry out functional annotation, whereas KEGG was used for pathway analysis. In parallel with this analysis, software from Thomson Reuters Corporation (https://portal.geneGO.com/) was used to further analyze the differentially expressed genes using Pathway Maps, GO Processes, Process Networks, Diseases (by Biomarkers), and Network Statistics.

qPCR was performed to confirm the observed changes in expression in the RNA-Seq analysis. Total RNA from THP-1 and THP-1-derived macrophages transfected with adenovirus US31 or control adenovirus was extracted with TRIzol reagent. RT-PCR was performed using a PrimeScript ${ }^{\mathrm{TM}}$ RT reagent kit (TaKaRa, Otsu, Japan) following the manufacturer's instructions. A customized PCR array from CT Bioscience ${ }^{37,38}$ (Changzhou, China) was used to compare the expression profile of a selected group of genes in the adenoviral US31 (Ad-US31)-transfected vs. Ad-GFP-transfected THP-1 
cells/THP-1-derived macrophages. Target mRNAs included molecules potentially involved in chemokine/cytokine signaling. PCR amplification of these cytokines/chemokines was performed for $10 \mathrm{~min}$ at $94{ }^{\circ} \mathrm{C}$, followed by 45 cycles of $94{ }^{\circ} \mathrm{C}$ for $15 \mathrm{~s}$, annealing at $60^{\circ} \mathrm{C}$ for $15 \mathrm{~s}$, and extension at $72{ }^{\circ} \mathrm{C}$ for $20 \mathrm{~s}$ in a Roche light cycler $480 \mathrm{II}$ PCR machine (Roche, Basel, Switzerland), using the SYBR Premix Ex Taq kit (TaKaRa). 32 -microglobulin (B2M), actin, beta $(A C T B)$, ribosomal protein L27 (RPL27), hypoxanthine phosphoribosyltransferase 1 (HPRT1), and ornithine decarboxylase antizyme $1(O A Z 1)$ were used as housekeeping genes for normalization. The relative quantitation method used was based on the $\mathrm{Ct}$ value of the test samples, and $2^{-\Delta \Delta C t}$ was used to express the relative expression of each cytokine/chemokine/receptor, whereby $\quad \Delta \Delta \mathrm{Ct}=\left(\mathrm{Ct}_{\text {cytokine/chemokine }}-\mathrm{Ct}_{\text {reference }}\right.$ gene $)$ experimental group $-\left(\mathrm{Ct}_{\text {cytokine/chemokine }}-\mathrm{Ct}_{\text {reference gene }}\right)$ control group. If the fold change $\left(2^{-\Delta \Delta \mathrm{Ct}}\right)$ was less than 1 , then then inverse $\left(-1 / 2^{-\Delta \Delta C t}\right)$ was used.

\section{Protein array screening of US31-binding proteins}

The Johns Hopkins Medical Institutions Protein Microarray Core produced the protein binding microarray chips with 19394 individual human GST-and His6tagged full-length proteins. Recombinant US31 purified from BL21/pET21a-US31 $(1.5 \mu \mathrm{g} / \mu \mathrm{l})$ was labeled using an Alexa-Flour-647 microscale protein labeling kit (A30009, Molecular Probes/Invitrogen). The data were extracted from the microarray images with GenePix ProTM 6.0. Background was defined as signals less than $20 \%$ of the maximum signal and removed from the subsequent analysis. The signal-to-noise ratios $(\mathrm{SNR}=\mathrm{F} 635$ median/ B635 median) were first calculated for all of the spots to generate the candidate list of US31-binding proteins. The SNR of a protein was calculated as the average of two duplicated spots.

\section{Bioinformatics analysis of the US31 interactome}

The PANTHER (Protein Analysis through Evolutionary Relationships) (http://www.pantherdb.org/) system, a unique resource that classifies genes and proteins according to their functions, was used to classify the US31 interactome. To evaluate protein overexpression, GO term enrichment, KEGG pathways, and Pfam domain families, DAVID 6.7 (https://david.ncifcrf.gov/) was used. The default human proteome was used as the background list. The $P$-value (EASE-score, Fisher-Pvalue, or Benjamini-Hochberg false discovery rate correction) indicates the significance of the pathway correlated with the conditions. The distribution of cellular components, molecular functions, and biological processes of the US31 interactome were also analyzed. The lower the $P$-value, the more significant the GO term $(P$-value $<0.05$ was considered statistically significant).

\section{Immunoprecipitation}

THP-1-derived macrophage cells were infected with the recombinant Ad-US31 or Ad-GFP control in 6-well dishes for $3 \mathrm{~h}$. The media was then replaced, and the cells were incubated as indicated. At $48 \mathrm{~h}$ post-transfection, the cells were lysed for $5 \mathrm{~min}$ on ice with immunoprecipitation (IP) buffer (Pierce) supplemented with cocktail protease inhibitors (Pierce). The lysates were then centrifuged to clear cell debris at $13,000 \times g$ for $10 \mathrm{~min}$ at $4{ }^{\circ} \mathrm{C}$. Then, $10 \mu \mathrm{g}$ of rabbit antibodies specific to US31, mouse antibodies specific to Flag (Sigma), or anti-NF-kB2 was combined with disuccinimidyl suberate binding to protein A/G plus agarose. Then, a Pierce ${ }^{\circ}$ Crosslink Immunoprecipitation Kit was used to collect the immune complexes as described in the manufacturer's protocol. The eluted protein complexes were then resuspended in reducing SDS sample buffer, heated at $95^{\circ} \mathrm{C}$ for $10 \mathrm{~min}$, and analyzed by immunoblotting.

\section{Ubiquitination assays}

For in vivo ubiquitination analysis, $30 \mu \mathrm{M}$ MG132 (Sigma- Aldrich) was applied to the cells. Ad-US31infected- THP1 derived macrophages were treated with MG132 starting at $36 \mathrm{hpi}$, and harvested at $42 \mathrm{hpi}$. DMSO was applied as the solvent control. Cell lysates were subjected to NF-кB2-directed IP (Cell Signaling Technology) followed by IB using a polyclonal rabbit antibody for ubiquitin (Cell Signaling Technology).

\section{Statistical analysis}

Data are expressed as the means \pm standard errors and statistical significance was tested using a t-test and ANOVA between the groups. Numerical data are represented as $n(\%)$ and statistical significance was tested using the chi-square test or Fisher's exact probability. A $P<0.05$ was considered statistically significant. All statistical results of the data were analyzed with SPSS 16.0 (Chicago, IL, USA).

\section{Acknowledgements}

This work was supported by the National Nature Science Foundation of China (Grant Nos. 81001343, 81472308 and 81672707), the Zhejiang Provincial Natural Science Foundation of China (grant no. Y2100909 and LY12H05003), Zhejiang Science and Technology Bureau (grant no. 2016C33214), and the Wenzhou Municipal Science and Technology Bureau (grant nos. Y20160018). We thank Jun Zhang for helpful suggestions in preparing the manuscript.

\section{Author details}

'Department of Microbiology and Immunology, Institute of molecular virology and immunology, Institute of Tropical Medicine, Wenzhou Medical University, Wenzhou, China. ${ }^{2}$ Second Clinical College, Wenzhou Medical University, Wenzhou, China. ${ }^{3}$ Department of Pathophysiology, Wenzhou Medical University, Wenzhou, China. ${ }^{4}$ Department of Laboratory Medicine, Second Affiliated Hospital \& Yuying Children's Hospital, Wenzhou Medical University, Wenzhou, China. ${ }^{5}$ Cardiac regeneration research institute, Wenzhou Medical University, Wenzhou, China. ${ }^{6}$ Department of Nephrology, First Affiliated Hospital, Wenzhou Medical University, Wenzhou, China 


\section{Authors' contributions}

GG: performed the experiments, analyzed and interpreted the data, and drafted the manuscript. SY, XS: performed the experiments and statistical analysis. SX, LY, CL, MY, BL, FW, ML, CC, LZ: acquired the data and material support. HZ: analyzed and interpreted the data, revised the manuscript and finally approved the version of the manuscript for publication. XX: made contribution to the conception and design, analyzed and interpreted the data, supervised the study, provided the project funding, revised the manuscript and finally approved the version of the manuscript for publication.

\section{Conflict of interest}

The authors declare that they have no conflict of interest.

\section{Publisher's note}

Springer Nature remains neutral with regard to jurisdictional claims in published maps and institutional affiliations.

\section{Supplementary information}

The online version of this article (https://doi.org/10.1038/s41419-017-0122-4) contains supplementary material.

Received: 1 August 2017 Revised: 28 October 2017 Accepted: 2 November 2017

Published online: 24 January 2018

\section{References}

1. Varani, S. \& Landini, M. P. Cytomegalovirus-induced immunopathology and its clinical consequences. Herpesviridae 2, 6 (2011).

2. Chen, J. et al. Correlation between systemic lupus erythematosus and cytomegalovirus infection detected by different methods. Clin. Rheumatol. 34, 691-698 (2015).

3. Guo, G. Q. et al. Different expressions of latent HCMV genes in UL133-UL138 locus was associated with systemic lupus erythematosus. Mol. Genet Microbiol + 32, 116-124 (2017).

4. Segal, B. H. \& Sneller, M. C. Infectious complications of immunosuppressive therapy in patients with rheumatic diseases. Rheum. Dis. Clin. North. Am. 23 219-237 (1997).

5. Zhang, Q. et al. Transcriptome altered by latent human cytomegalovirus infection on THP-1 cells using RNA-seq. Gene 594, 144-150 (2016).

6. Stevenson, E. V. et al. HCMV reprogramming of infected monocyte survival and differentiation: a Goldilocks phenomenon. Viruses 6, 782-807 (2014).

7. Chan, G., Bivins-Smith, E. R., Smith, M. S., Smith, P. M. \& Yurochko, A. D. Transcriptome analysis reveals human cytomegalovirus reprograms monocyte differentiation toward an M1 macrophage. J. Immunol. 181, 698-711 (2008).

8. Van Damme, E. \& Van Loock, M. Functional annotation of human cytomegalovirus gene products: an update. Front Microbiol 5, 218 (2014).

9. Van Damme, E. et al. HCMV Displays a Unique Transcriptome of Immunomodulatory Genes in Primary Monocyte-Derived Cell Types. PLOS. ONE. 11, e0164843 (2016).

10. Mukherjee, R. et al. Non-Classical monocytes display inflammatory features: Validation in Sepsis and Systemic Lupus Erythematous. Sci. Rep. 5, 13886 (2015).

11. Martinez, F. O., Gordon, S., Locati, M. \& Mantovani, A. Transcriptional profiling of the human monocyte-to-macrophage differentiation and polarization: new molecules and patterns of gene expression. J. Immunol. 177, 7303-7311 (2006).

12. Vallabhapurapu, S. \& Karin, M. Regulation and function of NF-kappaB transcription factors in the immune system. Annu. Rev. Immunol. 27, 693-733 (2009).

13. Brue, T. et al. Mutations in NFKB2 and potential genetic heterogeneity in patients with DAVID syndrome, having variable endocrine and immune deficiencies. Bmc. Med. Genet. 15, 139 (2014).

14. Manches, O., Fernandez, M. V., Plumas, J., Chaperot, L. \& Bhardwaj, N. Activation of the noncanonical NF-kappaB pathway by HIV controls a dendritic cell immunoregulatory phenotype. Proc. Natl. Acad. Sci. USA 109, 14122-14127 (2012).

15. Park, M. H. \& Hong, J. T. Roles of NF-kappaB in Cancer and Inflammatory Diseases and Their Therapeutic Approaches. Cells 5, E15 (2016).
16. De Silva, N. S. et al. Transcription factors of the alternative NF-kappaB pathway are required for germinal center B-cell development. Proc. Natl. Acad. Sci. USA 113, 9063-9068 (2016)

17. Vatsyayan, J., Qing, G., Xiao, G. \& Hu, J. SUMO1 modification of NF-kappaB2/ p100 is essential for stimuli-induced p100 phosphorylation and processing. Embo. Rep. 9, 885-890 (2008)

18. Goodrum, F. D., Jordan, C. T., High, K. \& Shenk, T. Human cytomegalovirus gene expression during infection of primary hematopoietic progenitor cells: a model for latency. Proc. Natl. Acad. Sci. USA 99, 16255-16260 (2002).

19. McCormick, A. L., Roback, L, Livingston-Rosanoff, D. \& St Clair, C. The human cytomegalovirus UL36 gene controls caspase-dependent and -independent cell death programs activated by infection of monocytes differentiating to macrophages. J. Virol. 84, 5108-5123 (2010).

20. Kwon, Y. et al. Inhibition of p53 transcriptional activity by human cytomegalovirus UL44. Microbiol. Immunol. 56, 324-331 (2012).

21. Rossetto, C. C., Tarrant-Elorza, M. \& Pari, G. S. Cis and trans acting factors involved in human cytomegalovirus experimental and natural latent infection of CD14 (+) monocytes and CD34 (+) cells. PLoS. Pathog. 9, e1003366 (2013).

22. Sharma, M., Kamil, J. P., Coughlin, M., Reim, N. I. \& Coen, D. M. Human cytomegalovirus $U L 50$ and $U L 53$ recruit viral protein kinase UL97, not protein kinase C, for disruption of nuclear lamina and nuclear egress in infected cells. J. Virol. 88, 249-262 (2014).

23. Borst, E. M. et al. The human cytomegalovirus UL51 protein is essential for viral genome cleavage-packaging and interacts with the terminase subunits pUL56 and pUL89. J. Virol. 87, 1720-1732 (2013).

24. Penkert, R. R. \& Kalejta, R. F. Tale of a tegument transactivator: the past, present and future of human CMV pp71. Future Virol. 7, 855-869 (2012).

25. Qian, Z., Xuan, B., Hong, T. T. \& Yu, D. The full-length protein encoded by human cytomegalovirus gene UL117 is required for the proper maturation of viral replication compartments. J. Virol. 82, 3452-3465 (2008).

26. Cheung, A. K, Abendroth, A., Cunningham, A. L. \& Slobedman, B. Viral gene expression during the establishment of human cytomegalovirus latent infection in myeloid progenitor cells. Blood 108, 3691-3699 (2006).

27. Burgdorf, S. W., Clark, C. L., Burgdorf, J. R. \& Spector, D. H. Mutation of glutamine to arginine at position 548 of IE2 86 in human cytomegalovirus leads to decreased expression of IE2 40, IE2 60, UL83, and UL84 and increased transcription of US8-9 and US29-32. J. Virol. 85, 11098-11110 (2011).

28. Basak, S., Shih, V. F. \& Hoffmann, A. Generation and activation of multiple dimeric transcription factors within the NF-kappaB signaling system. Mol. Cell. Biol. 28, 3139-3150 (2008)

29. Hochberg, M. C. Updating the American College of Rheumatology revised criteria for the classification of systemic lupus erythematosus. Arthritis Rheum. 40, 1725 (1997).

30. Bombardier, C., Gladman, D. D., Urowitz, M. B., Caron, D. \& Chang, C. H. Derivation of the SLEDAI. A disease activity index for lupus patients. The Committee on Prognosis Studies in SLE. Arthritis Rheum. 35, 630-640 (1992).

31. Daigneault, M., Preston, J. A., Marriott, H. M., Whyte, M. K. \& Dockrell, D. H. The identification of markers of macrophage differentiation in PMA-stimulated THP-1 cells and monocyte-derived macrophages. PLOS. ONE. 5, e8668 (2010).

32. GQ, G. et al. Constructing a recombinant adenovirus carying human cytomegalovirus UL138 gene and evaluating the effects of UL138 gene on the functions of monocytes. Chin. J. Microbiol. Immunol. 36, 667-675 (2016).

33. Zhu, S. et al. Peptide GC31 inhibits chemokines and ICAM-1 expression in corneal fibroblasts exposed to LPS or poly(l:C) by blocking the NF-kappaB and MAPK pathways. Exp. Eye. Res. 164, 109-117 (2017).

34. Ozcan, E. et al. Increased visfatin expression is associated with nuclear factorkappa B and phosphatidylinositol 3-kinase in periodontal inflammation. Clin. Oral. Investig. 21, 1113-1121 (2017).

35. Benedetti, F. et al. Sulfur compounds block MCP-1 production by Mycoplasma fermentans-infected macrophages through NF-kappaB inhibition. J. Transl. Med. 12, 145 (2014).

36. Zhang, Q. et al. Bioinformatics analysis to identify the critical genes, microRNAs and long noncoding RNAs in melanoma. Medicine 96, e7497 (2017).

37. Zhang, Y. W. et al. Integrated analysis of DNA methylation and mRNA expression profiling reveals candidate genes associated with cisplatin resistance in non-small cell lung cancer. Epigenetics 9, 896-909 (2014).

38. Zheng, Y. et al. Epigenetic downregulation of RUNX3 by DNA methylation induces docetaxel chemoresistance in human lung adenocarcinoma cells by activation of the AKT pathway. Int. J. Biochem. Cell. Biol. 45, 2369-2378 (2013).

39. Choi, W. S. et al. Structural basis for the recognition of $\mathrm{N}$-end rule substrates by the UBR box of ubiquitin ligases. Nat. Struct. Mol. Biol. 17, 1175-1181 (2010). 